\title{
Nanocarriers as pulmonary drug delivery systems to treat and to diagnose respiratory and non respiratory diseases
}

\author{
Malgorzata Smola ${ }^{1,2}$ \\ Thierry Vandamme' \\ Adam Sokolowski ${ }^{2}$ \\ 'Université Louis Pasteur, Faculté de \\ Pharmacie, Département de Chimie \\ Bioorganique, Illkirch Graffenstaden, \\ France; ${ }^{2}$ Wroclaw University of \\ Technology, Faculty of Chemical \\ Engineering, Wroclaw, Poland
}

\begin{abstract}
The purpose of this review is to discuss the impact of nanocarriers administered by pulmonary route to treat and to diagnose respiratory and non respiratory diseases. Indeed, during the past 10 years, the removal of chlorofluorocarbon propellants from industrial and household products intended for the pulmonary route has lead to the developments of new alternative products. Amongst these ones, on one hand, a lot of attention has been focused to improve the bioavailability of marketed drugs intended for respiratory diseases and to develop new concepts for pulmonary administration of drugs and, on the other hand, to use the pulmonary route to administer drugs for systemic diseases. This has led to some marketed products through the last decade. Although the introduction of nanotechnology permitted to step over numerous problems and to improve the bioavailability of drugs, there are, however, unresolved delivery problems to be still addressed. These scientific and industrial innovations and challenges are discussed along this review together with an analysis of the current situation concerning the industrial developments.
\end{abstract}

Keywords: nanotechnology, nanocarriers, nanoparticle, liposome, lung, pulmonary drug delivery, drug targeting, respiratory disease, microemulsion, bioavailability, micelle

\section{Introduction}

The removal of chlorofluorocarbon (CFC) propellants from industrial and household products, agreed by over 165 countries throughout the world, has lead to the developments of new alternative products. The timetable for this process is outlined in the Montreal protocol on substances that deplete the ozone layer document and in several subsequent amendments. These necessary changes offered the opportunity to the pharmaceutical companies and to research workers to develop new strategies to administer drugs by pulmonary route. These ones can be subdivided into two major parts, namely for the drugs intended on one hand for respiratory diseases and on the other hand for systemic diseases. In this second status, the lungs are only considered as a portal of entry for systemic drug delivery.

To replace CFC propellants, hydrofluoroalkanes (HFAs) are the new suggested alternative propellants for CFCs-12 and -114. Their potential for damage to the ozone layer is nonexistent, and while they are greenhouse gases, their global warming potential is a fraction (one-tenth) of that of CFCs. Replacement formulations for several inhalant respiratory medications have been or are being studied and tested, to assume the transition to these HFA or CFC-free pressurized metered dose inhalers (pMDIs) which will be complete by a near future. Currently, several CFC-free formulations are already available on the market throughout the world. Beside these new marketed devices, other comprising capsules containing powders called dry powder inhalers (DPIs) were also developed recently to avoid the use of chlorofluorocarbon propellants. 
To optimize the delivery of drugs, in this field, particular considerations have been focused on the forms (geometric design) of the particles, their physical states (amorphous or crystalline state) and their sizes ranging from the nanometric to the micrometric scale (Courrier et al 2002). In this field, it was also suggested to use the nanotechnology concept in order to increase the bioavailability of drugs administered by pulmonary route.

Face to these technological progresses, recently, advances have been also done in order to administer drugs via targeting dosage forms by pulmonary route for systemic applications. The conception of these new drug delivery systems (DDS) is to better control drug pharmacokinetics, pharmacodynamics, non-specific toxicity, immunogenicity and biorecognition of systems in the quest for improved efficacy. The rationale for the development of aerosol drug delivery systems for systemic delivery is based on the adoption of a multidisciplinary approach to the targeted delivery and release of drugs, underpinned by nanoscience and nanotechnology combining polymer science, pharmaceutics, bioconjugate chemistry and molecular biology. Therefore, this breakthrough is made possible by the development of various types of nanosystems resulting from cutting-edge researches based on pluridisciplinarity approaches. From the first immunoliposomes targeting to pulmonary endothelium proposed in 1990 by Maruyama et al (1990) and today, there was an explosion in the number of nanocarriers also called nanodevices or nanosystems which are defined as particles having a size ranging from $1 \mathrm{~nm}$ to $1 \mu \mathrm{m}$. To be suitable for pulmonary drug delivery, these ones are either made of lipids (liposomes, niosomes, microemulsions, lipidic micelles, solid lipid nanoparticles) or composed of polymers (polymer micelles, dendrimers, polymeric nanoparticles, nonogels, nanocapsules). These systems are exploited for therapeutic purpose to carry the drug in the body in a controlled manner from the site of administration to the therapeutic target. This implies the passage of the drug molecules and drug delivery system across numerous physiological barriers, which represent the most challenging goal in drug targeting.

In general nanocarriers aim to minimize drug degradation and loss, prevent harmful side effects and increase the availability of the drug at the disease site. Localized therapy of the target organ generally requires smaller total doses to achieve clinically effective results. To reach this goal, nanocarriers can be engineered to slowly degrade, react to stimuli and to be site specific. Targeting mechanisms can be either passive or active. An example of passive targeting is the preferential accumulation of chemotherapeutic agents in solid tumors as a result of the differences in the vascularization of the tumor tissue compared with healthy tissue. Active targeting involves the chemical "decorating" of the surface of drug carriers with molecules enabling them to be selectively attached to diseased cells. Nanosystems were also found useful to improve the performance of imaging techniques applied for the in vivo diagnosis of tumors. In this case, colloid metals are often incorporated in the nanodevice. Nanoscience and nanotechnology are thus the basis of innovative delivery techniques that offer great potential benefits to patients and new markets to pharmaceutical and drug delivery companies.

Aerosol administration of the therapeutics to the pulmonary epithelium for systemic delivery represents a significant opportunity for many classes of drugs and applications, including anti-tumor therapy, gene therapy, AIDS therapy, radiotherapy, in the delivery of macromolecules as peptides and proteins or small molecules as antibiotics, virostatics and vaccines. Advantages of aerosol administration include: (i) more rapid absorption into the systemic circulation (this may be specifically important for drugs where circulation (this may be especially important for drugs where fast onset of action is critical), and (ii) higher bioavailability than with other non-invasive modes of administration. This is certainly true for the delivery of peptides and proteins relative to oral administration, and is also true for many small molecules where first-pass metabolism or efflux transporters limit oral bioavailability.

\section{Anatomy and physiology of human respiratory tract}

The respiratory system works with the circulatory system to deliver oxygen from the lungs to the cells and remove carbon dioxide, and return it to the lungs to be exhaled. The exchange of oxygen and carbon dioxide between the air, blood and body tissues is known as respiration. Healthy lungs take in about 1 pint of air about $12-15$ times each minute. All of the blood in the body is passed through the lungs every minute. The respiratory tract is divided into two main parts: the upper respiratory tract, consisting of the nose, nasal cavity and the pharynx; and the lower respiratory tract consisting of the larynx, trachea, bronchi and the lungs (Figure 1). The trachea, which begins at the edge of the larynx, divides into two bronchi and continues into the lungs. The trachea allows air to pass from the larynx to the bronchi and then to the lungs. The bronchi divide into smaller bronchioles which branch in the lungs forming passageways for air. The terminal parts of the bronchi are the alveoli. The alveoli are the functional 


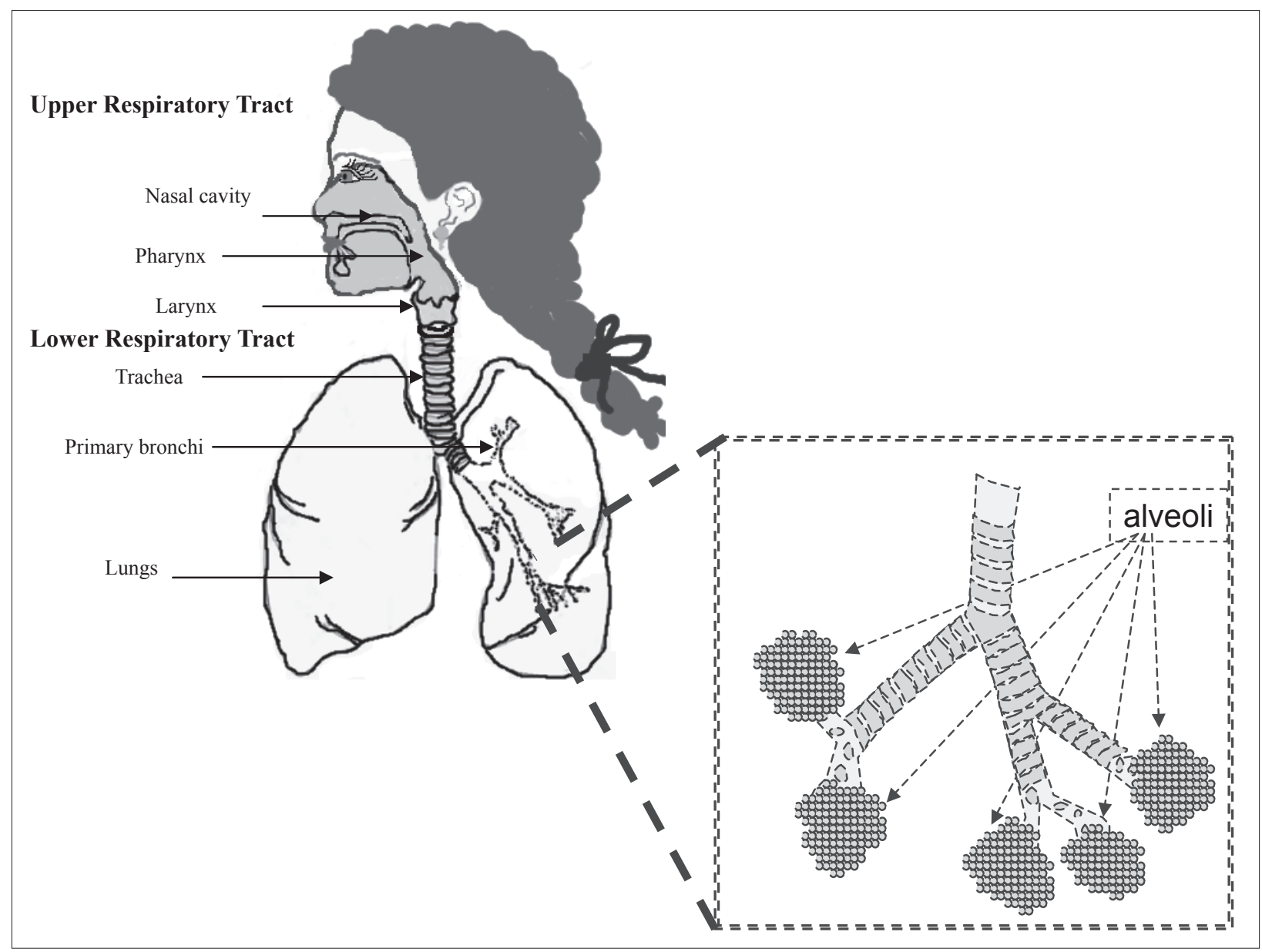

Figure I Regions of the respiratory system.

units of the lungs and they form the site of gaseous exchange (Marieb 2005).

\section{Alveoli}

The blood barrier between the alveolar space and the pulmonary capillaries is very thin to allow for rapid gas exchange. During inspiration, oxygen diffuses through the alveoli walls and the interstitial space, into the blood. Carbon dioxide diffuses in the opposite direction during exhalation. Alveoli are small and there are approximately 300 million of them in each lung. Although alveoli are tiny structures, they have a very large surface area in total $\left(\sim 100 \mathrm{~m}^{2}\right)$ for performing efficient gas exchange.

The alveoli form a honeycomb of cells around the spiral, cylindrical surface of the alveolar duct. The exposed alveolar surface is normally covered with a surface film of lipoprotein material.

There are several types of pulmonary alveolar cells. Type I (or small type A), are non-phagocytic, membranous pneumocytes. These surface-lining epithelial cells are approximately
$5 \mu \mathrm{m}$ in thickness and possess thin squamous cytoplasmic extensions that originate from a central nucleated portion. These portions do not have any organelles and hence they are metabolically dependent on the central portion of the cell. This reduces their ability to repair themselves if damaged.

Attached to the basement membrane are the larger alveolar cells (Type II, type B or septal cells). These rounded, granular, epithelial pneumocytes are approximately 10 to $15 \mu \mathrm{m}$ tick. There are 6 to 7 cells per alveolus and these cells possess great metabolic activity. They are believed to produce the surfactant material that lines the lung and to be essential for alveolar repair after damage from viruses or chemical agents (Washington et al 2001).

Amongst, the important roles of the lungs, one can cite: (i) supply oxygen, (ii) remove wastes and toxins, and (iii) defend against hostile intruders. The lungs have three dozen distinct types of cells. Some of these cells scavenge foreign matter. Others have cilia that sweep the mucous membranes lining the smallest air passages. Some cells act on blood pressure control, while others spot infection invaders. 
The respiratory system is susceptible to a number of diseases, and the lungs are prone to a wide range of disorders caused by genetic factors, infection and pollutants in the air. The most common problems of the respiratory system are:

- Asthma

- Bronchiolitis

- Chronic obstructive pulmonary disease (COPD)

- Common cold

- Cough

- Cystic fibrosis (CF)

- Lung cancer

- Pneumonia

- Pulmonary hypertension

- Respiratory diseases of newborns.

\section{Mechanism of deposition of particles and their becoming into the lungs after inhalation}

Aerosols are suspensions of solid or liquid particles in a gas (usually air). The particulate portion of an aerosol is referred to as particulate matter, or PM. Particulate matter is a generic term applied to chemically heterogeneous discrete liquid droplets or solid particles. The metric used for describing PM is the micron, or micrometer $\left(10^{-6}\right.$ meter $)$. The PM in an aerosol can range in size from 0.001 to greater than 100 microns in diameter. Particles intended to be administered by pulmonary route are generally categorized based on size:

- Coarse particles are larger than 2 microns in diameter

- Fine particles are between 0.1 and 2 microns in diameter

- Ultrafine particles are less than 0.1 micron

Most aerosol particles are polydisperse. They have a wide range of particle sizes that must be characterized by statistical measures. In some cases, such as with an ink jet printer, it is desirable to have a monodisperse aerosol with particles of equal size.

\section{Principal mechanisms of respiratory deposition}

The deposition of inhaled particles in the different regions of the respiratory system is very complex, and depends on many factors. Some of the factors influencing respiratory deposition include:

- Breathing rate

- Mouth or nose breathing

- Lung volume

- Respiration volume
- Health of the individual

- Bifurcations in the airways result in a constantly changing hydrodynamic flow field.

Depending on the particle size, airflow, and location in the respiratory system, particle deposition occurs via on of the following principal mechanisms:

\section{Impaction}

Each time the airflow changes due to a bifurcation in the airways, the suspended particles tend to travel along their original path due to inertia and may impact on an airway surface. This mechanism is highly dependent on aerodynamic diameter, since the stopping distance for very small particles is quite low. Impaction occurs mostly in the case of larger particles that are very close to airway walls, near the first airway bifurcations. Therefore, deposition by impaction is greatest in the bronchial region. Impaction accounts for the majority of particle deposition on a mass basis.

\section{Sedimentation}

Sedimentation is the settling out of particles in the smaller airways of the bronchioles and alveoli, where the air flow is low and airway dimensions are small. The rate of sedimentation is dependent on the terminal settling velocity of the particles, so sedimentation plays a greater role in the deposition of particles with larger aerodynamic diameters. Hygroscopic particles may grow in size as they pass through the warm, humid air passages, thus increasing the probability of deposition by sedimentation.

\section{Interception}

Interception occurs when a particle contacts an airway surface due to its physical size or shape. Unlike impaction, particles that are deposited by interception do not deviate from their air streamlines. Interception is most likely to occur in small airways or when the air streamline is close to an airway wall. Interception is most significant for fibers, which easily contact airway surfaces do to their length. Furthermore, fibers have small aerodynamic diameters relative to their size, so they can often reach the smallest airways.

\section{Diffusion}

Diffusion is the primary mechanism of deposition for particles less than 0.5 microns in diameter and is governed by geometric rather than aerodynamic size. Diffusion is the net transport of particles from a region of high concentration to a region of lower concentration due to Brownian motion. Brownian motion is the random wiggling motion of a particle due to the constant bombardment of air molecules. 
Diffusional deposition occurs mostly when the particles have just entered the nasopharynx, and is also most likely to occur in the smaller airways of the pulmonary (alveolar) region, where air flow is low.

\section{Absorption - bioavailability of drugs}

Although inhaled drugs have been used for over 50 years to treat airway disease and are in development or being considered for the treatment of many other lung diseases, insulin is at present time the only one representative inhaled drug on the market for systemic disease. Exubera ${ }^{\circledR}$ (insulin human [rDNA origin] inhalation powder is the first diabetes treatment which can be inhaled. Exubera ${ }^{\circledR}$ helps control high blood sugar, works in adults with type 1 diabetes and with type 2 diabetes as well (Adjei et al 1997). This therapeutic success has lead a number of other companies to investigate and to advance clinical trials as inhaled formulations for systemic applications with a variety of large molecules (leuprolide, a luteinizing hormone-releasing hormone (LHRH) analogue, ...) (Patton et al 1999). Recent advances in the development of particle technologies and devices now make it possible to formulate, stabilize, and accurately deliver almost any drug to the lungs.

The pulmonary membrane is naturally permeable to small molecule drugs and to many therapeutic peptides and proteins. The epithelium of the lung, the significant barrier to absorption of inhaled drugs, is thick $(50-60 \mu \mathrm{m})$ in the trachea, but diminishes in thickness to an extremely thin $0.2 \mu \mathrm{m}$ in the alveoli (Patton 1996). The change in cell types and morphology going from trachea, bronchi, and bronchioles to alveoli is very dramatic. The lungs are for more permeable to macromolecules than any other portal of entry into the body. Some of the most promising therapeutic agents are peptides and proteins, which could be inhaled instead of injected, thereby improving compliance (Patton 1996). Particularly, peptides that have been chemically altered to inhibit peptidase enzymes exhibit very high bioavailabilities by the pulmonary route (Schanker 1978). Indeed, natural mammalian peptides, les than 30 amino acids (somatostatin, vaso active intestinal peptide [VIP], and glucagons), are broken down in the lung by ubiquitous peptidases and have very poor bioavailabilities. Conversely, proteins with molecular weights between 6000 and $50,000 \mathrm{Da}$ are relatively resistant to most peptidases and have good bioavailabilities following inhalation (Patton et al 2004). For larger proteins, the bioavailabilities and absorption mechanisms are not well completely elucidated. For larger molecules such as proteins and peptides, airway epithelial junctions may be leakier than those in alveoli; the greater the fraction of drug deposited in the alveoli or deep lung, the higher the bioavailability for macromolecules like peptides or proteins (Patton et al 1999). Then, the absorption of exogenous macromolecules could be result from a combination of tight junctions and endocytic vesicles by processes that are diffusion-limited. There is evidence that for certain endogenous molecules that normally occur in lung lining fluids, eg, albumin, immunoglobulins and transferring, they are specific receptors mediated transport mechanisms on the alveolar epithelial cell that enable these proteins to be absorbed at higher rates than expected. For all days, plasma profiles resulting from inhaled drugs are a composite of drug absorbed across markedly different epithelia, and at this time it is very difficult to know what roles the different cells play in the absorption process. Although the pulmonary membrane is rich in antiproteases, aggregation of inhaled proteins will stimulate opsonization (coating) by special proteins in the lung lining fluids, which will then mark the aggregated proteins for phagocytosis and intracellular enzymatic destruction (Patton et al 2004). These enzymes are anchored in the plasma membranes of all cells and attack peptides at the ends of the amino acid chain, releasing one or two amino acids at a time. Proteins are usually poor substrates for peptidases, partly because the ends of their amino acid chains are often tucked into the globular structure of the protein and are not available for hydrolysis. In addition, the large size of proteins may preclude their fit into the catalytic clefts of the peptidase structures (Patton et al 2004). Thus, proteins can move about the body through the circulatory system and in the interstitum of tissues and remain "resistant" to peptidases that reside on the surfaces of all vessels, cells, and epithelial surfaces. Proteins are hydrolyzed by a different class of enzymes (ie, proteases), which are strictly controlled in the body and are located in specialized organelles (endosomes, lysosomes) (Patton 1996; Patton et al 2004).

Small peptides and proteins are absorbed more rapidly after inhalation than after subcutaneous injection. For other small molecules, inhalation is also a fast way to get into the body because drug efflux transporters and metabolizing enzymes are present in the lung at much lower levels than the gastrointestinal tract (Brown et al 1983; Colthorpe et al 1992; Niven et al 1994, Colthorpe et al 1995; Niven et al 1995). Lipophilic small molecules are absorbed extremely fast, $\mathrm{t}_{1 / 2}$ absorption being approximately $1-2$ minutes. Watersoluble small molecules are absorbed rapidly, $\mathrm{t}_{1 / 2}$ absorption being approximately 65 minutes. Small molecules can exhibit prolonged absorption if they are highly soluble or highly cationic (Niven et al 1994). 
Although the rapid absorption of molecules has many conceivable medical uses, there are situations when one might want to slow the absorption of an inhaled small molecule, either to keep it acting longer locally in lung, or to regulate its absorption into the body. Very insoluble molecules that slowly dissolve from the inhaled particle may stick in the lung for many hours or even days. Fluticasone propionate, amphothericin $\mathrm{B}$, and all-trans retinoic acid are absorbed from the lungs over a period of hours, due in part to their slow dissolution rate from relatively insoluble lipophilic particles (Brooks et al 2000; Beyer et al 1994). Encapsulation in slow release particles such as nanoparticles and liposomes can also be used to control absorption.

The bioavailability of peptides and proteins is 10-200 times greater by pulmonary route as compared with other non-invasive routes (Patton 1996). This fact can be attributed to the considerable absorptive surface at the air interface, covered by an extremely small volume of fluid (10-20 ml) and the entire cardiac output rushes through its capillary network, which lies fractions of a micron beneath the absorptive surface (Patton 1996). Due to this physiological and anatomical peculiarity, an inhaled aerosol can be widely dispersed and deposited in quite high concentrations in close proximity to the blood stream. Like the interior of the body, the surface fluids of the lung contain also antiproteases that inhibit the enzymatic breakdown of proteins. The release of proteases into the lung fluids by immune cells usually only occurs during infection and chronic inflammation which can cause the eventual destruction of the lung (such as with cystic fibrosis or emphysema). The high bioavailabilities of "blocked" peptides can be attributed either because they are linked into a ring like cyclosporine (and thus have no exposed ends) or they have chemical modifications of their $\mathrm{C}$ or $\mathrm{N}$ terminal ends which block peptidase attack (Patton et al 2004). Most of these peptides were derived from natural peptides that were too peptidase sensitive to make them useful therapeutic drugs. Clearly, susceptibility to enzymes is one of the most powerful determinants of a peptide's bioavailability through the lungs, and the use of blocking chemistry can make an ineffective peptide into one with high bioavailability and great medical value (Patton et al 2004).

One possible cause of low bioavailability of larger proteins is precipitation or aggregation in the alveolar lining fluid. As an aerosol particle settles in the lung, it contacts and is enveloped by at least a monolayer of lung surfactant (Patton 1996). Any aggregates of undissolved protein will be opsonized (coated) with various immune and non immune proteins that mark a particle for macrophage ingestion that, in the case of proteins, usually means enzymatic degradation. Because larger proteins are slowly absorbed by the lungs, they have a long time to clump and aggregate and are cleared by alveolar macrophages. Lombry et al demonstrated that a primary source of elimination of inhaled macromolecules after delivery to the lungs and before absorption into the systemic circulation owed to clearance by alveolar macrophages (AM). Therefore, this fact may have important ramifications on the development of inhalation as an attractive mode of administration of therapeutic proteins to the blood stream.

Before discussing the interest of nanotechnology applied to deliver a drug efficiently into the lungs, it is important to mention some practical issues with inhalation delivery. First of all, to reach the alveoli, the drug must be inhaled as particles with aerodynamic diameters somewhere between 1 and $3 \mu \mathrm{m}$. The way the patient inhales, the form and the size of the particles constituting the aerosol will have a considerable impact on the eventual primarily deposition in the conducting airways (trachea, bronchi, and bronchioles) or in the alveoli. Large aerosol particles are not absorbed intact since they must dissolve before to be absorbed. Material that does not reach the lungs is deposited in the device or oropharynx of the patient.

\section{Significance of nanotechnology by pulmonary route}

Pulmonary drug delivery offers local targeting for the treatment of respiratory diseases and increasingly appears to be a viable option for the delivery of drugs systemically. Pulmonary delivery is carried out in a variety of ways - via aerosols, metered dose inhaler systems, powders (dry powder inhalers) and solutions (nebulizers), which may contain nanostructures such as micelles, liposomes, nanoparticles, and microemulsions. Research into lung delivery is driven by the potential for successful protein and peptide drug delivery by this route and by the promise of an effective delivery mechanism for gene therapy (eg, in the treatment of cystic fibrosis) (Okumura et al 1997), as well as the need to replace chlorofluorocarbon propellants in metered dose inhaler systems. However, the success of pulmonary delivery of protein drugs is diminished by proteases in the lung, which reduce their overall bioavailability, and by the barrier between capillary blood and alveolar air (the air-blood barrier). Therefore, adjuvants such as absorption enhancers and protease inhibitors are sometimes required to enhance the pulmonary absorption of such macromolecular or poorly absorbable drugs. Niven and Byron reported that adjuvants such as oleic acid, oleyl alcohol, and Span 85 can increase 
the transfer rate of disodium fluorescein in isolated rat lungs (Niven and Byron 1990). Yamamoto et al (1997) reported that the pulmonary absorption of insulin was enhanced in the presence of various adjuvants such as glycocholate, surfactin, Span 85, and nafamostat. They reported also that $n$-lauryl $\beta$-D-maltopyranoside (LM), linoleic acid-HCO60 (hydrogenated caster oil) mixed micelles (MM), and sodium glycocholate (NaGC) all effectively enhanced the pulmonary absorption of fluorescein isothiocyanate dextrans (FDs) and insulin. These adjuvants did not cause severe mucosal damage of rat lung, as determined by the leakage of Evans blue from the systemic circulation (Yamamoto et al 1997). These findings suggested that the use of absorption enhancers and protease inhibitors would be a useful approach for improving the pulmonary absorption of biologically active drugs like peptides. Furthermore, besides the importance of the choice of excipients to increase the pulmonary absorption of drugs, the design (size, shape, and aerodynamic properties) of the dosage forms (nanocarriers) can contribute also to the improvement of the treatment.

\section{Micelles}

A successful drug carrier system needs to demonstrate optimal drug loading and release properties, long shelf-life and low toxicity. Colloidal systems, such as micellar solutions, vesicle and liquid crystal dispersions, as well as nanoparticle dispersions consisting of small particles of 10-400 nm diameter show great promise as carriers in pulmonay drug delivery systems. Drugs can be trapped in the core of a micelle and transported at concentrations even greater than their intrinsic water solubility. A hydrophilic shell can form around the micelle, effectively protecting the contents. In addition, the outer chemistry of the shell may prevent recognition by the reticuloendothelial system, and therefore early elimination from the bloodstream. A further feature that makes micelles attractive is that their size and shape can be changed. Chemical techniques using crosslinking molecules can improve the stability of the micelles and their temporal control. Micelles may also be chemically altered to selectively target a broad range of disease sites.

Amphiphilic macromolecules self-assemble to nanoscopic core/shell structures in an aqueous environment, namely polymeric micelles that can encapsulate water insoluble drugs, proteins and DNA, and target therapeutics to their site of action in a passive or active way. Polymeric micelles share many structural and functional features with natural transport system, eg, virus and lipoproteins. The versatility of the synthetic chemistry in polymeric micelles is a bonus providing opportunities to design appropriate nano-carriers for individual delivery requirements. Development of nanoengineered polymeric micellar formulations that can address the problem of drug resistance correspond to one focus of this drug targeting dosage form. In this context, the effect of chemical manipulations on the encapsulation, release, bio-distribution and cellular interaction of the polymeric nanocarrier is assessed to select appropriate chemistries for optimized delivery of P-glycoprotein substrates to resistant tumors. Another focus of this nanometric dosage form is to improve the solubilization of a poorly water soluble drug intented to be administered via pulmonary route.

The advantages of polymeric micelles include better stability than surfactant micelles, ability to solubilize an adequate amount of hydrophobic drugs, prolonged circulation times in vivo, and capability to accumulate in the target organs. In this field, Jones and Leroux designed beclomethasone dipropionate-loaded polymeric micelles directly administrable to the lung in nanoparticle sizes in inhalation dosage form intended to be an effective means of treating asthma and chronic pulmonary obstructive disease. This concept was able to be achieved since the polymeric micelles are able to evade the mononuclear phagocytic system due to their bulky hydrophilic outer shell and sustain the release of the drug (Marsh et al 2003). In addition, drug loaded polymeric micelles are strongly suggested to pass through the mucus layer associated with bronchial inflammatory diseases directly to their receptors in the epithelial cells. For these reasons, the polymeric micelles are very beneficial in delivering hydrophobic corticosteroids, such as beclomethasone dipropionate, for the treatment of chronic pulmonary obstructive disease since the inability of such drugs to penetrate through the mucus layer to reach the target site, largely the result of failure in treatment in the past. In addition, it was also reported that a mammalian secreted phospholipase A2 (PLA2) was able to degrade pegylated phosphatidyl ethanolamine (Davidsen et al 2001; Vermehren et al 2001). This finding supported the potential of pulmonary delivery micelles to target glucocorticosteroids to the inflammed tissues of the lung.

In this context, Gaber et al evaluated the potential of using poly-(ethylene oxide)-block-distearoyl phosphatidylethanolamine (mPEG-DSPE) polymer to prepare beclomethasone dipropionate loaded micelles with high entrapment efficiency and mass median aerodynamic diameter of less than $5 \mu \mathrm{m}$, and demonstrated their sustained release properties. The entrapment efficiency of beclomethasone dipropionate, effects of drug-polymer molar ratio on particle size, physicochemical properties, in vitro inhalation pattern, and in vitro 
drug release profile of polymeric micelles were also investigated by the same authors and allowed them to conclude of the therapeutic interest of this kind of nanocarriers.

\section{Liposomes}

The utilization of liposomal drug formulations for aerosol delivery has many potential advantages, including aqueous compatibility, sustained pulmonary release to maintain therapeutic drug levels and facilitated intra-cellular delivery particularly to alveolar macrophages (Schreier et al 1993). Furthermore, drug-liposomes may prevent local irritation and reduce toxicity both locally and systematically (Schreier et al 1993; Gonzalez-Rothi and Schreier 1995). Increased potency with reduced toxicity is characteristic of many drug-liposomal formulations (Cullis et al 1989). Liposomal aerosols (including CsA) have proven to be non-toxic in acute human and animal studies (Thomas et al 1991; Myers et al 1993; Waldrep, Arppe, et al 1997; Waldrep, Gilbert, et al 1997). These results suggest that drug-liposome aerosols should be more effective for delivery, deposition and retention of water-insoluble, hydrophobic, lipophilic compounds in contrast to water soluble compounds (Niven and Schreier 1990; Taylor et al 1990; Taylor and Farr 1993). The goal of targeted drug-liposomal aerosol therapy is to maximize delivery and retention while minimizing clearance. Studies of liposomal CsA have demonstrated a propensity for bilayer membrane exchange both in vitro and in vivo (Choice et al 1995; Fahr et al 1995; Ouyang et al 1995).

The development of liposomal formulations for aerosol delivery with jet nebulizers has expanded the possibilities for effective utilization of aerosol based therapies in the treatment of pulmonary diseases. The property of sustained release or depot effect of liposomes has been studied using different tracer molecules to monitor absorption and clearance of liposomes from the lung. A cyclosporin A (CsA)-dilauroylphosphatidylcholine (DLPC) liposomal formulation for aerosol delivery to the lung has been studied by Waldrep, Nieminen, et al (1997). These authors determined by HPLC the pharmacokinetics of both CsA and liposomal carrier (labeled with ${ }^{99 \mathrm{~m}}$ technetium $\left({ }^{99 \mathrm{~m}} \mathrm{Tc}\right.$ )) to study potential dissociation after delivery to normal mouse lungs. Same authors compared also the effects of pulmonary inflammation on the clearance of CsA-DLPC liposomes with 99mTc labeled human serum albumin (HSA). Results indicated that $99 \mathrm{mTc}$-DLPC liposome carrier is retained up to 16.9 times longer than the CsA half-life in normal lung and 7.5 times longer in inflamed lungs. Similar values were obtained also for $99 \mathrm{mTc}$-labeled albumin (14.8 times for normal CsA half life (6.8 times in inflamed lungs)) (Arppe et al 1998)

The development of liposomal formulations, compatible with aerosol delivery with jet nebulizers, has also expanded the possibilities for more effective utilization of aerosol based therapies for the treatment of a variety of pulmonary diseases (Farr et al 1985; Gilbert et al 1988; Niven and Schreier 1990; Schreier et al 1993; Taylor and Farr 1993). Such utilization of liposomes, as aerosol delivery vehicles, has many reported potential advantages for clinical development, including: aqueous compatibility facilitated intra-cellular delivery particularly to alveolar macrophages and lymphocytes and sustained pulmonary release to maintain therapeutic drug levels within the lung (Schreier et al 1993; Taylor and Farr 1993; Hung et al 1995). These factors, however, have not been extensively studied in the human lung.

Human studies of liposome aerosols have demonstrated similar retention within the normal lung, but, depending on the presence of inflammation (Vidgren et al 1994; Saari et al 1998). Factors affecting the clearance and/or elimination of exogenous materials from normal and diseased lung tissues are not yet completely understood. The mucociliary clearance system is impaired in the diseased lung (Schreier et al 1993), including asthma (O'Riordan et al 1992). In contrast, experimental studies lead in rats with infection and/or associated pulmonary inflammation have showed enhanced clearance of instilled drug-liposomes and inhaled ${ }^{99 m}$ Tc-HSA (Connelly and Peterson 1993; Omri et al 1994). Thus, multiple factors could also influence drug-liposome retention times within the lung.

Using gamma scintigraphy, nebulizer systems were evaluated and selected for optimal aerosol targeting of drug-liposomes to the peripheral lung regions (Vidgren et al 1994). However, recent studies of CsA-liposomes in other experimental systems have reported that CsA displays a unique property of rapid bilayer membrane exchange (Choice et al 1995; Fahr et al 1995; Ouyang et al 1995). The aerosol particle sizes of these labeled liposomes was 1.54 (1.64) mm

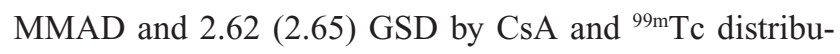
tion, respectively. The mean particle size of the liposomes in solution by Nicomp analysis was $564 \mathrm{~nm}$. The clearance studies of ${ }^{99 \mathrm{~m}} \mathrm{Tc}$-labelled CsA-DLPC liposomes delivered to the lungs of normal Balb/c mice demonstrated that there was an apparent dissociation between CsA and ${ }^{99 \mathrm{~m}} \mathrm{Tc}$-labelled DLPC molecule independent of inflammation similarly as it was previously described for intravenous liposomal CsA by Choice et al (1995), Fahr et al (1995), and Ouyang et al 
(1995). Therefore Arppe et al (1998) concluded that CsA clearance from the lung was unaffected by inflammation.

Studies of pharmacokinetics lead by Arppe et al (1998) demonstrated that the ${ }^{99 \mathrm{~m}}$ Tc-DLPC (1,2 dilauroyl-sn-glycero3-phospholine) is retained up to 16.9 times longer than the pulmonary half lives of CsA $\left(\mathrm{T}_{1 / 2}\right)$ in normal lung ( 7.5 times in inflamed lung). The pulmonary half lives of $\operatorname{CsA}\left(\mathrm{T}_{1 / 2}\right)$ in this system was unaffected by pulmonary inflammation with concurrent lymphocytic infiltration and cyclophilin binding proteins. While the CsA-DLPC liposomes used in this study were extremely stable in vitro, the results suggested that there was dissociation mediated by multiple uncharacterized factors in the lung following pulmonary delivery of CsA-DLPC. This in vivo dissociation phenomenon did not, however, preclude the delivery of immunosuppressive activities to the lung and thus, CsA-liposome aerosol therapy may be utilized in the treatment of chronic bronchiolar asthma and a variety of pulmonary diseases, such as pulmonary sarcoidosis and allergic hypersensitivity.

Nebulization of rehydrated freeze-dried beclomethasone dipropionate liposomal preparations showed that the output of liposomes from the nebulizer was dependent on the lipid used (Darwis and Kellaway 2001). Liposomes formed from lipids with a high $\mathrm{Tm}$ exhibited a low output of aerosols, due to aggregation during nebulization (Darwis and Kellaway 2001). To compare beclomethasone dipropionate liposomal formulations, the same drug encapsulated in poly(L-lactic acid) yielded microspheres in aerosolizable particle size range of less than $5 \mu \mathrm{m}$. Nevertheless, degradation of these microspheres could only release $15 \%$ of the drug over a period of eight days (El-Baseir et al 1997). As a result of nanoprecipitation, anhydrous beclomethasone dipropionate in beclomethasone dipropionate-poly(L-lactic acid) nanoparticles was changed to beclomethasone dipropionate monohydrate (Hyvönen et al 2005). Also, recently, non-phospholipid vesicles loaded with beclomethasone dipropionate were fabricated with non-ionic surfactant, polysorbate 20 (Terzano 2005). Even though the fine particle fraction (FPF) with aerodynamic diameter of less than $5 \mu \mathrm{m}$ was $95 \%$ and the permeation rate through model mucosal barrier was greatly enhanced, the maximum entrapment efficiency was only $20 \%$.

Shahiwala and Misra compared bioavailabilities of different pulmonary formulations intended to provide prolonged effective concentration of levonogestrel in plasma and to reduce reported side effects of orally administrated drug. Levonogestrel encapsulated liposomes containing $10 \mu \mathrm{g}$ of drug were instilled intratracheally in rats and were compared with the plain drug suspension and the physical mixture also administrated by the same route (plain drug with liposomal constituents). The plasma drug concentration data of different treatements were plotted and pharmacokinetics data were calculated and compared with that of oral administration. Percentage relative bioavailability of $97.6 \%, 98.6 \%$, and $109.9 \%$ were observed after pulmonary administration of plain drug formulation, physical mixture (plain drug along with constituents of liposomes), and liposomal formulations of the drug, respectively. Following oral administration, $\mathrm{C}_{\max }$ of $14.4 \pm 0.6 \mathrm{ng} / \mathrm{mL}$ was observed at $2.1 \pm 0.2$ hours followed by subtherapeutic concentration beyond $30 \pm 0.2$ hours, while after pulmonary administration of formulations, $\mathrm{C}_{\max }$ of 4.4 $\pm 0.4 \mathrm{ng} / \mathrm{mL}, 4.2 \pm 0.5 \mathrm{ng} / \mathrm{mL}$, and $4.4 \pm 0.6 \mathrm{ng} / \mathrm{mL}$ were observed at $6.0 \pm 0.2$ hours, $7.0 \pm 0.2$ hours, and $6.8 \pm 0.2$ hours, respectively, followed by maintenance of effective plasma drug concentration up to $60 \pm 2$ hours. The studies lead by Shahiwala and Misra (2004) allowed to demonstrate on one hand superiority of pulmonary drug delivery with regards to maintenance of effective therapeutic concentration of the levonorgestrel in the plasma over a period of 6 to 60 hours and on the other hand to reduce frequency of dosing and systemic side effects associated with oral administration of levonorgestrel.

Stern et al (2000) advanced the potential of liposomes as gene carriers. These authors demonstrated that the pretreatment with cationic lipid-mediated transfer of the $\mathrm{Na}+$ $\mathrm{K}+$-ATPase pump in a mouse model in vivo augmented resolution of high permeability pulmonary oedema. This demonstration of a significant reduction in pulmonary edema following in vivo gene transfer allowed raising the possibility of gene therapy as a novel localized approach for pulmonary edema in clinical settings such as acute respiratory distress syndrome (ARDS) and lung transplantation (Stern et al 2000).

\section{Microemulsions}

Likewise the micellar solutions, the emulsions and microemulsions are dosage forms showing numerous advantages providing that the surfactants used are not toxic. Anyway, more and more exogenous surfactants, used for treatments and as a precaution for acute respiratory distress syndrome (ARDS), are used as solutions or suspensions drug targeting systems. Therefore, these ones allow envisaging at once a respiratory treatment and a drug delivery system. These surfactants are considered as effective drug targeting systems if they don't interfere with the therapeutic activity of the drug. Indeed, incorporation of a drug into micellar solutions 
constituted with Cremophor ${ }^{\circledR}$ EL increases its bioavailability until 77\% (Taljanski et al 1997).

Very few emulsions or microemulsions have been studied to administer drugs by the pulmonary route (Lawrence and Rees 2000). However, these dosage forms show numerous advantages compared to other drug targeting systems: easiness to be manufactured and maximum of drug to be incorporated. Indeed, the drug being soluble into one phase, this one will be located preferentially into this phase, leading to an encapsulation close to $100 \%$. Due to their physicochemical characteristics, reverse emulsions and microemulsions should allow to solubilize a large amount and a lot of hydrophilic drugs.

Several aerosol formulations designed with an external phase constituted of a propellant have been described. As example, one can cite water-in-chlorofluorocarbon reverse micelles including proteins and peptides stabilized by lecithin (Evans and Farr, 1993). Even if this dosage form is stable and is able to deliver peptides and proteins efficiently by pulmonary route, its use should be limited since the Montreal protocol (1987) recommended stopping the production and the use of chlorofluorocarbons. Propellants like hydrofluoroalkanes (HFAs) or propane have been suggested. Reverse microemulsions stabilized by lecithin and using propane and dimethylether as propellants have been also described (Sommerville et al 2000). These microemulsions, characterized by mean geometric diameters ranged between 1 and $5 \mu \mathrm{m}$ and by a respirable fraction up to $36 \%$, showed high stability during more than 4 weeks at room temperature. Water-in-HFA emulsions stabilized by non ionic fluorinated surfactants have been also studied in order to administer drugs by pulmonary route (Patel et al 1998). Butz et al (2002) and Courrier et al (2004) studied new reverse miniemulsions and microemulsions based on fluorinated surfactants intended for pulmonary delivery of drugs. Miscibility behavior studies of dipalmitoylpho sphatidylcholine with a single-chain perfluoroalkylated amphiphile in Langmuir monolayers indicated that the (perfluorooctyl)undecyldimorpholinophosphate surfactants softened the lipid, which may be crucial for the performance of this material during the dimensional changes of the monolayer imposed during inhalation-exhalation cycles (Courrier, Vandamme et al 2003; Hiranita et al 2003). The evaluation of cytotoxicity of new semi-fluorinated amphiphiles derived from dimorpholinophosphate allowed to conclude that semifluorinated compounds with the highest fluorinated chain length/total chain length ratio were the least toxic (Courrier, Krafft et al 2003; Courrier 2004). Moreover, reverse water-in-fluorocarbon emulsions stabilized with these amphiphiles were found to be non-cytotoxic, or less cytotoxic than solutions of the same amphiphiles in fluorocarbons. Administered by intranasal route to mice, those emulsions didn't alter the lungs and didn't induce lung inflammatory reactions, but they induced a body weight decrease which was found to be reversible. These reverse water-in-fluorocarbon emulsions showed their potential as lung delivery system by the recorded hypoglycemic effect after the intranasal administration of those emulsions incorporating insulin (Courrier et al 2004).

\section{Micro- and nanoparticles}

The terminology "microparticle" (size comprised between 1 and $999 \mu \mathrm{m}$ ) includes the microspheres (uniform sphere constituted of a polymeric matrix) and the microcapsules (container constituted of an oily core surrounded by a thin polymeric membrane).

Biodegradable microspheres, designed from natural or synthetic polymers, have been largely used as drug targeting systems via different routes. Hydrophilic and lipophilic molecules can be encapsulated or incorporated into microspheres. Compared to liposomes, microspheres have an in vivo and in vitro more stable physicochemical behaviour and should allow a slower release and a longer pharmacological activity of the encapsulated drugs. Biodegradable microspheres are prepared by using varied polymers: albumin, chiotosan, polysaccharide (Illum, 1997), poly(lactic-co-glycolic) acid, poly(lactic) acid, poly(butylcyanoacrylate) and poly(lacticco-lysine graft lysine).

Pulmonary administration of aerosolized microspheres allows a sustained and prolonged release of drugs for respiratory or non respiratory diseases, in this last case, the drug being protected against the enzymatic hydrolysis. Microspheres can be produced following different requirements such as the morphology, the size and the porosity by varying different technological parameters during their preparation. Microspheres are less hygroscopic and are then less liable to swell in the presence of moisture located into the lungs (El Baseir and Kellaway 1998).

Studies relative to pulmonary targeted dosage forms using encapsulated drugs into microspheres have been developed recently. Different drugs have been encapsulated into microspheres including corticosteroids (El Baseir and Kellaway 1998), viruses (Greenway et al 1995; Eyles et al 2000), proteins (Morimoto et al 2000), tuberculostatics (Suarez et al 2001; Zhang et al 2001), and anticancer agents (Mu and Feng 2002; Feng and Chien 2003). 
Chitosan-microspheres have been formulated in order to be spread out into hydrofluoroalkanes (HFAs), novel propellant used for pressurized inhalers having a respirable fraction ranging up to $18 \%$ (Williams et al 2000).

Nanoparticles present the same characteristics than the microspheres: they are also constituted of polymers or lipids and drugs bound either at the surface of the particles either encapsulated into the vector. In this last case, a protection against the enzymatic degradation and a modified bioavailability of the drug can be envisaged and increased by a controlled release. These targeting systems (Kawashima et al 1998, 1999; Takeuchi et al 2001) can be designed for in vivo applications including molecules with therapeutic activities and radiocontrast agents or in vitro as a support for molecules intended for diagnosis. Manufacturing and encapsulating methods for drugs and the feasibility of modifying the surfaces of these vectors have been reviewed by different authors (Couvreur et al 2006; Lemarchand et al 2006). Drug targeting studies using these vectors by pulmonary route have been essentially conducted by encapsulating insulin (Kawashima et al 1999; Zhang et al 2001).

Large porous PLGA-particles $\left(\rho \sim 0.4\right.$ g.cm $\left.{ }^{-3}, d>5 \mu \mathrm{m}\right)$ showed to be more efficient for the pulmonary drug delivery of inhaled particles than small porous or not porous particles ( $\rho \sim 1 \pm 0.5$ g.cm $\left.{ }^{-3}, d<5 \mu \mathrm{m}\right)$ (Edwards et al 1997). This efficacy was attributed to the fact that large particles aggregate less and deaggregate more easily under shear forces than smaller, non-porous particles - all other considerations equal (French et al 1996; Li et al 1996) - hence they appear to more efficiently aerosolize from a given inhaler device than conventional therapeutic particles.

Very few particles composed of biocompatible and biodegradable polymers have been studied for the targeting of drugs by pulmonary route (Zeng et al 1995; Li et al 2001). Synthetic polymers are much more frequently used than natural polymers. Even if the synthetic polymers are well tolerated as implants, they are not biologically inert when they are not biologically inert when they are used as particles (Armstrong et al 1996). In fact, particles having sizes ranging from nanometers to micrometers can be internalized by macrophages and then degraded inside the cells, leading to a possible toxicity. Then, these solid polymeric particles can induce a cytotoxic (Müller et al 1996) or inflammatory effect (Armstrong et al 1996).

The size of particles plays a crucial role for the appearance of inflammatory symptoms. Indeed, ultrafine polystyrene particles $(\sim 60 \mathrm{~nm})$ showed much more developed pro-inflammatory activities than nanoparticles having a size ranged between 200 and $500 \mathrm{~nm}$ and this, due to the much more pronounced importance of their specific area (Brown et al 2001).

Since 1991, solid lipid nanoparticles (SLN) have been developed in order to design alternative colloidal drug targeting particles to liposomes and polymeric nanoparticles (Müller et al 2000). Indeed, SLN combine the advantages of the biocompatibility of lipids and the possibility of industrial scale up of nanoparticles. Like for polymeric micro- and nanoparticles, three possible loadings of drugs can be envisaged: (i) dispersion of drugs into the particle; (ii) core-membrane model containing a membrane improved with the drug; (iii) core-membrane model containing a core improved with the drug. Already, drugs like prednisolone, diazepam and camptotecin have been incorporated into SLN for pulmonary applications (Müller et al 2000). The key parameters determining the encapsulation rate of drugs into lipids are: (i) the solubility of the drug into melt lipids, (ii) the miscibility of the melt drugs into melt lipids, (iii) chemical and physical structures of the lipid solid matrix, and (iv) the polymorphous state of the lipids. In vitro release studies have showed that an encapsulated drug into solid lipid nanoparticles can diffuse during a period of time ranging from 5 to 7 weeks. Also, dispersions of solid lipid nanoparticles can be spray dried without significant change of the sizes of the particles.

Others nanoparticles, based on lecithin, have been also studied in order to ensure their dispersion into hydrofluoroalkanes (HFAs) to generate aerosolization properties (Dickinson et al 2001). In this study, the respirable fraction ranged up to $88 \pm 8 \%$. These nanoparticles showed an ideal deposition profile of inhaled particles intended for systemic delivery of drugs.

Following the interesting results obtained at the outcome of the studies lead on porous and lipid particles, Pulmospheres $^{\mathrm{TM}}$ have been made up (Dellamary et al 2000). These particles are made of phospholipids $\left(\rho<0.2 \mathrm{~g} . \mathrm{cm}^{-3}\right.$, $2.3<\mathrm{d}<4.5 \mu \mathrm{m}$ ) easily to be dispersed into HFAs, from their sizes ranging between 50 and $300 \mathrm{~nm}$ and having the advantage to release uniform amounts of drugs when they are aerosolized. A prolonged release of immunoglobulins incorporated into these microparticles has been studied (Bot et al 2000).

\section{Cyclodextrins}

Cyclodextrins (CDs) are the result of the association of oligosaccharides and are formed of six, seven or eight units of glucopyranose ( $\alpha-, \beta$ - or $\gamma-C D$, respectively). The outside 
part of CDs has hydroxyl groups which arehydrophobic whereas the inner part is relatively lipophilic. Following the complete or partial inclusion of the drug into the cavity, the drug can interact by non covalent bondings with CDs, becoming higher soluble in an aqueous medium (Duchêne, Ponchel et al 1999; Duchêne, Wouessidjewe et al 1999).

$\beta-C D$ seems to be the more used cyclodextrin for pharmaceutical development, due to the size of its cavity, the complexation efficiency with drugs and their relatively low production costs. CDs have been studied to encapsulate drugs and to be used in this application to target drugs into the lungs. CDs are able to complex testosterone, salbutamol or rolipram (Rajewski and Stella 1996). Cyclodextrins can be used also in combination with other vectors. They are able to increase the encapsulation rate of drugs into microparticles and to modulate their releases (Duchêne et al 1999). Principally, they were described and used by pulmonary route for their pulmonary absorption promoter of peptides and proteins (Sayani and Chien 1996) like insulin (Chetty and Chien 1998) or calcitonin (Kobayashi et al 1996).

CDs exert a relatively mild and reversible effect on the ciliary beat frequency of both chiken embyo trachea and human nasal adenoid tissue in vitro in a concentration-dependent manner (Sayani and Chien 1996; Irie and Uekama 1997).

\section{Optimization of the pulmonary vector}

A stable formulation of the drug targeting system depends of several factors: (i) the composition and the structure of the vector, (ii) the manufacturing process, (iii) the nature and the proportion of the incorporated drug. The size of the vector is a key parameter, in particular for the pulmonary administration. The optimum formulation of the vector corresponds to (i) a maximum efficiency of the incorporation of the drug, (ii) an efficiency of the administration which allows the best distribution of the drug and to generate the best therapeutic activity.

\section{Protection of the pharmacological activity of the drug}

The encapsulated drugs into the vectors are protected against the degradation or the transformation attributable to the external pulmonary medium. As example, the anticancer drug 9-nitrocamptothecin (9NC) has been encapsulated into DLPC-liposomes in which the lactone for of the camptothecin has been preserved in the liposomes during pulmonary deposition, even in the presence of albumin (Knight et al
2000). In fact, albumin combines with the camptothecin carboxyl form, involving an almost total loss of 9-NC anticancer activity. The degradation of salmon calcitonin by the enzymes associated to the pulmonary membranes seems also to be avoided by encapsulating the drug into microspheres made of gelatine (Morimoto et al 2000).

Similarly, PLGA-microspheres can protect plasmids from nuclease degradation and therefore offer an effective approach for in vivo gene delivery especially to phagocyte cells, for inducing immunization. The dissolution of the drug into the pulmonary mucus is avoided if the drug is encapsulated into the vector. For example, this is the case of encapsulated corticosteroids into PLA or PGLA microspheres.

\section{Decrease of the systemic toxicity of drug}

The encapsulation of drug into vectors avoids the local irritations and decreases the local and systemic toxicity of the drug. Moreover, the targeted pulmonary administration of the vector allows decreasing the amount of the encapsulated drug, since the total amount of drug is released locally. The amount of effective 9NC contained in the liposomes is 10-50 times lower than that used by other routes of administration (Knight et al 2000). The greater therapeutic effectiveness is a result of rapid absorption in the respiratory tract and, more specifically, in the pulmonary tissues, and penetration into the organ and tumor sites. Other studies (Koshkina et al 2000) were investigated with 9NC-liposomes, by atomizing them into mice with pulmonary metastases caused by B16 melanoma or human osteosarcoma. In both cases, the administration of 9NC-liposomes in aerosol form led to a reduction in pulmonary weight and number and size of metastases. Treatment with 9NC-liposomes appeared to be effective against pulmonary tumors.

\section{Effeciency of encapsulation}

Physicochemical characteristics of the drug

By choosing the drug targeting system, the physicochemical characteristics of the drug is crucial in order to allow a satisfactory encapsulation rate. Ionic drugs are usually used to be dissolved into the aqueous phase. The drug to be encapsulated into the liposomes has to be hydrophilic and water-soluble in order that the membrane of the liposome can be used for keeping the therapeutic molecule inside of the cavity and allows a prolonged release (Gonzales-Rothi et al 1996).

Liposomes of EYPC-cholesterol (CHOL) incorporating dexamethasone palmitate (DEXP), in a molar proportion of 4:3:0.3 were studied (Benameur et al 1995). Encapsulation of 
the DEXP was effective (70\%) in comparison with its nonesterified form $(<2 \%)$. The biological activity of DEXP was evaluated on blood mononuclear cells over a 24-hour period, measuring its anti-lymphocyte proliferation properties and its inhibition of interferon- $\gamma$ production (Table 1). The DEXP incorporated into the liposomes kept its biological activity. Nebulization studies in animals should confirm whether this vector was promising in drug delivery to the lungs.

On the contrary, a lipophilic drug is preferably used to be encapsulated into polymeric microspheres because of its homogenous dispersion into the polymeric matrix. As example, beclomethasone dipropionate (BDP) showed an encapsulation rate of $88 \%$ into PLA microspheres whereas sodium nedocromil was encapsulated only with a rate of $9 \%$ (El-Baseir et al 1998).

\section{Physicochemical characteristics of the vector}

Reasonable encapsulation rates, between $10 \%$ and $35 \%$ can be obtained during the formulation of liposomes (GonzalezRothi et al 1996; Suntres and Shek 1998). The physicochemical characteristics of the drug targeting system have to be adequately selected so that to have a maximum efficiency of the encapsulation of the drug. Partially negative charged liposomes allow an efficient encapsulation (70\%) of cationic peptides (Lange et al 2001). Also, Ten et al (2002) reported that DMPC-liposomes allowed to encapsulate $98.8 \%$ of Interleukin 2 (IL-2).

Concerning the polymeric microspheres, the encapsulation rates are generally higher $(20 \%-50 \%)$ principally due to the existence of different technologies allowing the encapsulation of a drug. However, the molecular weight of the polymers can play on the efficiency of the encapsulation of the drug. Indeed, when the PLGA has a molecular weight of $12,500 \mathrm{~g} \cdot \mathrm{mol}^{-1}$, the encapsulation efficiency for DNA reaches $30.0 \%$ whereas a molecular weight of 50,000 g.mol ${ }^{-1}$ allowed an encapsulation rate of $53.3 \%$ (Wang et al 1999).

\section{Drug delivery systems without encapsulation}

Kanaoka et al (1999) showed that the presence of empty liposomes can also stabilize nebulized INF- $\gamma$. INF- $\gamma$ nebulized alone is unstable, with these two cysteines producing intra- and intermolecular bonds then involving polymerization and aggregation. This method has the advantage of avoiding the incorporation of INF- $\gamma$ in the liposomes as well as separating free INF- $\gamma$ and liposomes. The liposome size remained identical before and after atomizing. Because they are unilamellar (UL) vesicles, these liposomes were too small and too rigid to be deformed. The size of the nebulized droplets was
Table I Inhibition (\%) of concavalin A stimulating proliferation of lymphocytes and production of interferon $\gamma(\text { INF- } \gamma)^{a}$

\begin{tabular}{lll}
\hline $\begin{array}{l}\text { Inhibition \% } \\
\text { concavalin A-stimulating }\end{array}$ & Free DEXP & Liposome-DEXP \\
\hline Lymphocytes proliferation & 94 & 94 \\
INF- $\gamma$ production & 96 & 96 \\
\hline
\end{tabular}

ainduced by 10-6 M of free dexamethasone palmitate (DEXP) or by DEXP loaded liposomes composed of EPC-Cholesterol. (Benameur et al 1995).

identical with or without liposomes. Therefore, liposomes do not interfere in the delivery of INF- $\gamma$. It was calculated that approximately 100 liposomes were combined with a molecule of INF- $\gamma$.

DPPC-CHOL (7:2) liposomes encapsulating insulin of various oligomerization degrees were instilled by the i.t. route in rats (Liu et al 1993). These studies revealed that only the initial response (10 $\mathrm{min})$ of encapsulated hexameric insulin is slower than that of dimeric insulin, suggesting a slower permeability through the pulmonary epithelium. However, the hypoglycemic effect was identical for both encapsulated oligomers, as it was for the physical mixture of insulin and blank liposomes. Prolonged absorption of insulin is not due to encapsulation but to the liposome surface connection and probably to an interaction between the exogenous DPPC and pulmonary surfactant.

Complexes between drugs and vectors are more and more designed, especially in gene therapy. Cationic liposomes are in particular used to complex DNA. Certain drugs have been spray dried successfully and showed the expected therapeutic activity, namely the proteinic activity.

\section{Increase of the pulmonary retention and bio-activity}

At same concentration, an encapsulated drug into a vector is held during a longer time inside the lungs than the same drug in solution. Then, the kinetic profile of the therapeutic activity of the drug is prolonged when this one is encapsulated into the vector. In this case, the controlled release of the drug allows the decrease of the side effects due to the removal of the first-pass metabolism of the drug, less administrations of dosage forms and a best compliance of the patient.

It has been showed that a half-hour after the instillation, the pulmonary concentration of glucocorticoids was twice higher when the drug is encapsulated into liposomes compared to the solubilized drug (Suntres and Shek 1998). The therapeutic activity was significantly prolonged during more than 72 hours whereas the free glucocorticoids didn't 
show any therapeutic activity after 24 hours. Similar results have been observed by using encapsulated antibiotics into liposomes (Demayer et al 1993).

The pulmonary retention of the vector has also to be studied: it allows to determine the kinetic release of the drug from the vector. Generally, the drug is eliminated more rapidly than the vector. As example, liposome vectors and cyclosporine A (CsA) dissociation were studied in mice following pulmonary delivery (Arppe et al 1998).

A stable radioactive complex of ${ }^{99 m}$ Tc-liposomes DLPC/ CsA was delivered by intratracheal (i.t.) instillation. The ${ }^{99 \mathrm{~m}} \mathrm{Tc}$-liposomes DLPC vector was retained 17 times longer than the half-life of CsA in a normal lung and 7.5 times longer than in an inflamed lung.

\section{Determination of the kinetic drug release}

Generally, the vectors are used to determine the kinetic release of the drug inside of the lungs. The encapsulated drug into the vector shows a therapeutic activity which is copared to the same drug in solution, at the same concentration. The kinetic release of the drug depends essentially of the composition and of the structure of the vector.

By using liposomes, the kinetic profile of the drug is depending of the glass temperature $\left(\mathrm{Tg}{ }^{\circ} \mathrm{C}\right)$ of the lipids used to formulate the liposomes. A high glass temperature means that the liposomes will be rigid and the speed of the drug release will be slow. For this reason, liposomes composed of DPPC $\left(\mathrm{Tg} \sim 42{ }^{\circ} \mathrm{C}\right)$ will release a drug more slowly than liposomes composed of DLPC $\left(\mathrm{Tg} \sim-2^{\circ} \mathrm{C}\right)$ or of EPC $\left(-10 \pm 5^{\circ} \mathrm{C}\right)$. Incorporation of cholesterol (CHOL) into the mixture of lipids will make rigid the structure (= membrane) of liposomes. A formulation of liposomes composed principally of DPPC and negative charged lipids like dimyristoylphosphatidylglycerol (DMPG) $\left(\mathrm{Tg} \sim 23{ }^{\circ} \mathrm{C}\right.$ ) allowed a prolonged and sustained release of the encapsulated antibiotics during more than 48 hours. The physical interaction of a lipophilic drug and the lipids constituting the bilayer of liposomes will lead to a decrease of the Tg of the lipids used (Darwis and Kellaway 2001). Microparticles containing the same drug but constituted of different polymers showed different kinetic releases of the encapsulated drug. As example, PLGA microspheres (MW $\sim 15,000$ ) released $20 \%$ of a drug after 8 hours whereas PLA-microspheres (MW 2,000) containing the same drug released this one completely after the same time. The kinetic release of the drug is also depending of charges present at the surface of the particles and principally the electrostatic difference between the drug and the molecules constituting the vector, leading then to an attraction or repulsion. Indeed, after 2 hours, $85 \%$ of salmon calcitonin are released from positive charged microspheres whereas $40 \%$ were released from negative charged microspheres (Morimoto et al 2000).

\section{Therapeutic requirements}

The design of a formulation can be the reflection of a therapeutic requirement. Indeed, for a formulation to be spray dried, the atomizing time has to be reduced to reach its maximum in order to favor the compliance of the patient by keeping the therapeutic activity. In this last case, a formulation incorporating a maximum of drug is then wished. To respond to these requirements, liposomes of DLPC containing concentrated amounts of CsA and budesonide (Bud) have been formulated and nebulized (Waldrep, Nieminen et al 1997). Formulations 40 times more concentrated than commercial ones and used by nebulization of Bud suspensions could both reduce nebulization time and improve patient compliance.

\section{Cellular adhesion}

\section{Target or avoid alveolar macrophages?}

Targeting drugs to alveolar macrophages has the distinct advantage of delivering high concentrations of drug to a cell that plays a central role in the progression of disease (tuberculosis) and in immune responses.

The microspheres can target alveolar macrophages (AMs) without eliciting a pulmonary inflammatory response in vitro ( $\mathrm{Ng}$ et al 1998). In fact, a cell culture of $\mathrm{AM}$, in the presence of microspheres composed of PLA, produces negligible quantities of oxidants and tumor necrosis factor alpha (TNF- $\alpha$ ) inflammatory cytokines. Interactions between PLA microspheres, marked by rhodamine $6 \mathrm{G}$, which is a fluorescent agent, and AM, are concentration-dependent $(\sim 30 \%$ interactions with a concentration of 50,000 particles $/ \mathrm{mL}$ ). Endocytosis of the microspheres was revealed in the presence of certain endocytosis inhibitors - lysosomotropic agents, $\mathrm{NH}_{4} \mathrm{Cl}$, and chloroquine - reducing AM - particle interaction by around $50 \%$. This study demonstrated that microspheres can enter alveolar macrophages without activating them, thus enabling possible drug delivery to target macrophages, for example, in the case of tuberculosis.

Liposomes, due to their similar compositions of the pulmonary surfactant can interact with life cells like macrophages by different ways, namely: adsoption, endocytocis, exchange of lipids or fusion. 


\section{Mucoadhesive systems}

Bioadhesive drug delivery systems were introduced in order to prolong and intensify the contact between controlled delivery forms and the mucous apical pole, inducing active transport processes (Lehr 2000). Contact with the mucus of the epithelium is called muco-adhesion, and direct contact with the cellular membrane is called cyto-adhesion. Lectins are nonimmunological glycoproteins that have the capacity to recognize and bind to glycoproteins exposed at the epithelial cell surface.

Liposomes functionalized with lecithins appeared to be capable of improving their binding to human alveolar cells (A549 and primary cells) (Abu-Dahab 2001). In this study, the unfunctionalized liposome formulation was optimized by measuring the loss of carboxyfluorescein (CF) loaded in the liposomes during atomization. Liposomes composed of DPPC-CHOL $(50 \%-50 \% \mathrm{~mol})$ were more stable during atomization ( $8 \% \mathrm{CF}$ loss) than DPPC liposomes (15\%-20\% CF loss), even in the presence of pulmonary surfactant. Lehr (2000) reported that the atomization of DPPC-CHOL liposomes with lecithin functionalization did not significantly influence their physical stability. The cell-binding capacity of functionalized liposomes is much higher than that of unfunctionalized liposomes, even after atomization

Mucoadhesive nanoparticles, coated with mucoadhesive polymers such as poly(acrylic acid) or chitosan, were aerosolized in guinea pigs via the trachea (Takeuchi et al 2001). Chitosan-modified nanospheres (CS-nanospheres), with a diameter of around 700-800 $\mathrm{nm}$, demonstrated a slower elimination rate, about half that observed with unmodified nanospheres. These results indicate that CS-nanospheres adhere to the mucus in the trachea and in the lung tissues as a result of the mucoadhesive properties of chitosan and release the drug in the lung over a prolonged period of time. The bioactivity of encalcotin encapsulated in CS- and unmodified nanospheres were compared with the bioactivity of elcatonin in solution $(100 \mathrm{IU} / \mathrm{kg})$ after aerosolization. Unmodified nanospheres and the drug solution induced a temporary fall in blood calcium levels after administration, returning to normal after 8 hours, whereas CS-nanospheres induced a significantly prolonged reduction in blood calcium lasting over 24 hours. It is believed that the unmodified nanospheres are rapidly eliminated from the lung before they are able to release the drug. The prolonged pharmacological effects of CS-nanospheres may be attributed to their adherence to lung tissue, meaning that they remain there for a considerable period of time. These results show that mucoadhesive nanospheres may be useful for the pulmonary delivery of peptide drugs.

Mucoadhesive microspheres of hydroxypropylcellulose (HPC) encapsulating beclomethasone dipropionate (BDP) were administered as powder aerosols to healthy or asthmatic guinea pigs (Sakagami et al 2001). The pharmacokinetics and pharmacodynamics of BDP were compared for different BDP formulations: pure crystalline BDP (cBDP), amorphous BDP incorporated in HPC microspheres (aBDP-HPC), and crystalline BDP-loaded HPC microspheres (cBDP-HPC). Powder aerosols were produced within a respirable size range of 1.7-2.9 $\mu \mathrm{m}$. The pharmacokinetic profiles for these three powders were dissolution modulated. It was shown that at 180 minutes postadministration, more than $95 \%$ and $85 \%$ of BDP were absorbed from the lung following aBDP-HPC and cBDP administration, respectively; whereas $86 \%$ of BDP were absorbed at 180 minutes following cBDP-HPC administration. Prolonged lung retention of BDP may be beneficial in maximizing the efficacy of BDP dose delivery to the lung and in reducing the side effects caused by its extra lung absorption. The duration of inhibition of eosinophil infiltration into the airways of asthma-induced guinea pigs was assessed following cBDP and cBDP-HPC administration. While cBDP $\left(1.37 \mathrm{mg} \cdot \mathrm{kg}^{-1}\right)$ inhibited eosinophil infiltration for only 1-6 hours, cBDP-HPC, with a lower drug dosage $\left(0.25 \mathrm{mg} \cdot \mathrm{kg}^{-1}\right)$, was able to maintain these inhibitory effects for 24 hours following administration. This study showed that this HPC microsphere system has the potential to prolong the therapeutic duration of BDP following inhalation.

\section{Immunospecific systems}

Immunoliposomes - liposomes carrying specific antibodies can target cells carrying a specific antigen. Margalit (1995) reported that they have been used to target pulmonary tumors in vitro and in vivo.

\section{Conclusion and perspectives}

As discussed in this review, the pulmonary drug delivery field is truly one of the most up-and-coming areas in today's applied pharmaceutical research. The reason is not because of the recent launch of inhaled insulin but the fact that pulmonary delivery still offers many white areas on the map. Indeed, still at this time, the more information is collected the more related question marks are surfacing covering the area of lung physiology and diseases, lung deposition, intelligent inhalation devices, delivery of biopharmaceuticals, absorption enhancement, controlled drug release in the lung and, last but not least, the topic of vaccination of 
the lung. From the examples exposed in this review, it can be concluded that, at present time, it is indeed possible to some extend to deliver larger drug molecules such as insulin and heparin systematically up to therapeutic drug levels.

Several reasons can be advanced to justify the long time which was necessary for the first pulmonary product to reach market approval.

First of all the physiology of the lung is not designed to absorb drug compounds systemically despite the very fragile and vulnerable nature of the epithelium. As an interface between the body's circulation and the outside air its function is to exchange only gas molecules and it has built up a strong defense against any other components that may enter the lung by accident. The defense can be by either physical by mucosal clearance or physiological by a strong presence of macrophages and enzymes. The lung can also develop immunological reactions against unwanted entities such as viruses. Secondly, a drug molecule needs to pass the physical clearance system represented by the upper airways and reach preferably the deep lung. Thirdly, once deposited in the lung periphery, it should avoid the biological defense mechanism and be absorbed by the alveolar epithelium. Consequently, in order to reach the lung periphery as efficiently as possible, several state-of the art devices haven been developed. The most prominent ones in the case of insulin are developed by Nektar Therapeutics (Exubera ${ }^{\circledR}$ inhaler) and Aradigm (AERx), where a defined particle cloud is generated which can be efficiently administered through slow inhalation by the patient. Besides these ones, other intelligent inhalation devices were also developed like the Akita technology. Akita is equipped with a "smartcard" that contains the patient's physiological lung function data so that the inhalation maneuver can be adapted to the individual lung. Last year a pediatric device was also introduced to the market that translates the electronically controlled features of the Akita device very nicely by mechanical means. In this case, the inhalation volume is defined by the size of the balloon in the device chamber and the inhalation velocity is controlled by the air-inlet valve at the back of the device. The aerosol can be nebulized into the device by, for example, any commercially available metered dose inhaler (MDI) system that is connected to the mouthpiece of the device. In line with the most recent European regulations that emphasize the need to design suitable application tools for children, this is certainly one of the inventive highlights in the pulmonary field besides the launch of Exubera ${ }^{\circledR}$.

What can be expected in the near future? Which conclusions can be drawn from the past experimental facts?
In spite of the numerous developments of nanocarriers intended for pulmonary drug delivery, today it appears more and more that the use of controlled drug releasing systems in the lung is a controversial issue. Beyond academic attempts, not many commercial solutions have emerged so far. The major reason is the highly efficient physiological defense system for particulates in the lung that is difficult to overcome. As soon as a foreign particle is observed in the lung tissue a powerful macrophage reaction is launched to attack the particle. Probably, the most promising approaches involve the use of stealth approaches, such as PEGylation or the use of endogenous compounds that occur in the lung such as dipalmitoylphosphatidylcholine (DPPC). DPPC can be coformulated into liposomes that encapsulate a drug compound for prolonged release as shown recently in an interesting study based on these findings and using the AeroLEF system (aerosolized Liposomal Encapsulated Fentanyl).

Another future interesting application of the pulmonary route is the delivery of vaccines. Besides providing a convenient alternative to needle injections it also has shown to be more efficient in antibody responses than injected vaccine in a recently published article by Bennett et al (2002). This concept would really benefit compliance of patients that are otherwise difficult to reach. In particular, in economically less fortunate regions in the world where infectious diseases are strong threats to quality of life and one of the major causes of mortality, vaccination programs by inhalation can be regarded as a clear advantage of invasive administration. Due to its interesting potential, this area will be another hot topic in the field of pulmonary drug delivery for the coming 10 years.

\section{References}

Abu-Dahab R, Schäfer UF, Lehr C-M. 2001. Lectin-functionalized liposomes for pulmonary drug delivery: effect of nebulization on stability and bioadhesion. Eur J Pharm Sci, 14:37-46.

Adjei LA, Gupta PK. 1997. Inhalation delivery of therapeutic peptides and proteins. In Lenfant C, ed. Lung biology in health and disease. New York: Marcel Dekker, Inc. p 1-913.

Armstrong DJ, Elliott PNC, Ford JL, et al. 1996. Poly - (D,L - lactic acid) microspheres incorporating histological dyes for intra-pulmonary histopathological investigations. J Pharm Pharmacol, 48:258-62.

Arppe J, Vidgren M, Waldrep JC. 1998. Pulmonary pharmacokinetics of cyclosporin A liposomes. Int J Pharm, 161:205-14.

Benameur H, Latour N, Schandene L, et al. 1995. Liposome-incorporated dexamethasone palmitate inhibits in-vitro lymphocyte response to mitogen. J Pharm Pharmacol, 47:812-17.

Bennett JV, de Castro JF, Valdespino-Gomez JL, et al. 2002. Aerosolized measles and measles-rubella vaccines induce better measles antibody booster responses than injected vaccine: randomized trials in Mexican school children. Bulletin of the World Health Organisation, 80:806-12.

Beyer J, Schwartz S, Barzen G, et al. 1994. Use of amphotericin B aerosols for the prevention of pulmonary Aspergillosis. Infection, 22:143-8. 
Bot AL, Tarara TE, Smith DJ, et al. 2000. Novel lipid-based hollow-porous microparticles as a platform for immunoglobulin delivery to the respiratory tract. Pharm Res, 17:275-83.

Brooks AD, Tong W, Benedetti F, et al. 2000. Inhaled aerosolization of alltrans-retinoic acid for targeted pulmonary delivery. Cancer Chemother Pharmacol, 46:313-18.

Brown DM, Wilson MR, MacNee W, et al. 2001. Size-dependent proinflammatory effects of ultrafine polystyrene particles: a role for surface area and oxidative stress in the enhanced activity of ultrafines. Toxicologly Applied Pharmacol, 175:191-9.

Brown RA Jr, Schanker LS. 1983. Absorption of aerosolized drugs from the rat lung. Drug Metab Disp, 11:355-60.

Butz N, Porté C, Courrier H, et al. 2002. Reverse water-in-fluorocarbon emulsions for use in pressurized metered-dose inhalers containing hydrofluoroalkane proppellants. Int J Pharm, 238:257-69.

Chetty DJ, Chien YW. 1998. Novel methods of insulin delivery: an update. Crit Rev Ther Drug Carrier, 15:629-70.

Choice E, Masin D, Bally MB, et al. 1995. Liposomal cyclosporine. Comparison of drug and lipid carrier pharmacokinetics and biodistribution. Transplantation, 60:1006-11.

Colthorpe P, Farr SJ, Smith IJ, et al. 1995. The influence of regional deposition on the pharmacokinetics of pulmonary-delivered human growth hormone in rabbits. Pharm Res, 12:356-9.

Colthorpe PS, Farr SJ, Taylor G, et al. 1992. The pharmacokinetics of pulmonary delivered insulin: a comparison of IT and aerosol administration to the rabbit. Pharm Res, 9:764-8.

Connelly JC, Peterson BT. 1993. Clearance of Tc-labeled albumin from lungs in anesthetized guinea pigs. Exp Lung Res, 19:237-55.

Courrier HM, Butz N, Vandamme ThF. 2002. Pulmonary drug delivery systems: recent developments and prospects. Crit Rev Ther Drug Carrier Syst, 19:425-98.

Courrier HM, Krafft MP, Butz N, et al. 2003. Evaluation of cytotoxicity of new semi-fluorinated amphiphiles derived from dimorpholinophosphate. Biomaterials, 24:689-96.

Courrier HM, Pons F, Lessinger JM, et al. 2004. In vivo evaluation of a reverse water-in-fluorocarbon emulsion stabilized with a semifluorinated amphiphile as a drug delivery system through the pulmonary route. Int J Pharm, 282:131-40.

Courrier HM, Vandamme TF, Krafft MP. 2004. Reverse water-influorocarbon emulsions and microemulsions obtained with a fluorinated surfactant. Colloids and Surface A: Physicochemical and Engineering Aspects, 244:141-8.

Courrier HM, Vandamme TF, Krafft MP, et al. 2003. Mixed monolayers made from dipalmitoylphosphatidylcholine and a fluorinated amphiphile. Colloids and Surface A: Physicochemical and Engineering Aspects, 215:33-41.

Couvreur P, Vauthier C. 2006. Nanotechnology: intelligent design to treat complex disease. Pharm Res, 23:1417-50

Cullis PR, Mayer LD, Bally MB, et al. 1989. Generating and loading of liposomal systems for drug delivery systems. Adv Drug Deliv Rev, 3:267-82.

Darwis Y, .Kellaway IW. 2001. Nebulization of rehydrated freeze dried beclomethasone dipropionate liposomes. Int J Pharm, 215:113-21.

Davidsen J, Vermehren C, Frokjaer S, et al. 2001. Drug delivery by phospholipase A2 degradable liposomes. Int J Pharm, 214:67-9.

Dellamary LA, Tarara TE, Smith DJ, et al. 2000. Hollow porous particles in metered dose inhalers. Pharm Res, 17:168-74.

Demaeyer P, Akodad EM, Gravet E, et al. 1993. Disposition of liposomal gentamicin following intrabronchial administration in rabbits. $J$ Microencapsul, 10:77-88.

Dickinson PA, Howells SW, Kellaway IW. 2001. Novel nanoparticles for pulmonary drug administration. J Drug Targeting, 9:295-302.

Duchêne D, Ponchel G, Wouessidjewe D. 1999. Cyclodextrins in targeting. Application to nanoparticles. Adv Drug Del Rev, 36:29-40.

Duche^ne D, Wouessidjewe D, Ponchel G. 1999. Cyclodextrins and carrier systems. J Control Rel, 62:263-8.
Edwards DA, Hanes J, Caponetti G, et al. 1997. Large porous particles for pulmonary drug delivery. Science, 276:1868-71.

El-Baseir MM, Kellaway IW. 1998. Poly(lactic acid) microspheres for pulmonary drug delivery: release kinetics and aerosolization studies. Int J Pharm, 175:135-46.

El-Baseir MM, Phipps MA, Kellaway IW. 1997. Preparation and subsequent degradation of poly(L-lactic acid) microspheres suitable for aerosolization: a physicochemical study. Int J Pharm, 151:145-53.

Evans RM, Farr SJ. 1993. Aerosol formulations including proteins and peptides solubilized in reverse micelles and process for making the aerosol formulations. US005230884A. UK.

Eyles JE, Williamson ED, Spiers ID, et al. 2000. Protection studies following bronchopulmonary and intramuscular immunisation with yersinia pestis FI and V subunit vaccines coencapsulated in biodegradable microspheres: a comparison of efficacy. Vaccine, 18:3266-71.

Fahr A, Holz M, Fricker G. 1995. Liposomal formulations of cyclosporin A: Influence of lipid type and dose on pharmacokinetics. Pharm Res, 12:1189-98.

Farr SJ, Kellaway IW, Parry-Jones DR, et al. $1985 .{ }^{99 \mathrm{~m}}$ Technetium as a markers of liposomal deposition and clearance in the human lung. Int J Pharm, 26:303-16.

Feng S-S, Chien S. 2003. Chemotherapeutic engineering: Application and further development of chemical engineering principles for chemotherapy of cancer and other diseases. Chem Engin Sci, 58:4087-114.

Feng SS, Mu L, Chen BH, et al. 2002. Polymeric nanospheres fabricated with naturel emulsifiers for clinical administration of an anticancer drug paclitaxel (Taxol®). Mat Sci Eng, C 20:85-92.

French DL, Edwards DA, Niven RW. 1996. The influence of formulation on emission, deaggregation and deposition of dry powders for inhalation. J Aerosol Sci, 27:769-83.

Gaber NN, Darwis Y, Peh K-K, et al. 2006. Characterization of polymeric micelles for pulmonary delivery of beclomethasone dipropionate. J Nanosci Nanotechnol, 6:3095-101.

Gilbert BE, Six HR, Wilson SZ, et al. 1988. Small particle aerosols of enviroxime-containing liposomes. Antiviral Res, 9:355-65.

Gonzalez-Rothi RJ, Schreier H. 1995. Pulmonary delivery of liposomeencapsulated drugs in asthma therapy. Clin Immunother, 4:331-7.

Gonzales-Rothi RJ, Suarez S, Hochhaus G, et al. 1996. Pulmonary targeting of liposomal triamcinolone acetonide phosphate. Pharm Res, 13:1699-703.

Greenway TE, Eldridge JH, Ludwig G, et al. 1995. Induction of protective immune responses against Venezuelan equine encephalitis (VEE) virus aerosol challenge with microencapsulated VEE virus vaccine. Vaccine, 16:1314-23.

Hiranita T, Nakamura S, Kawachi M, et al. 2003. Miscibility behavior of dipalmitoylphosphatidylcholine with a single-chain partially fluorinated amphiphile in Langmuir monolayers. $J$ Colloid and Interface Sci, 265:83-92.

Hung OR, Whynot SC, Varvel JR, et al. 1995. Pharmacokinetics of inhaled liposome-encapsulated fentanyl. Anesthesiology, 83:277-84.

Hyvönen S, Peltonen L, Karjalainen M, et al. 2005. Effect of nanoprecipitation on the physicochemical properties of low molecular weight poly(L-lactic acid) nanoparticles loaded with salbutamol sulphate and beclomethasone dipropionate. Int J Pharm, 295:269-81.

Illum L. 1997. Polysaccharide microspheres for the pulmonary delivery of drugs: WO 971/35562.

Jones MC, Leroux JC. 1999. Polymeric micelles. A new generation of colloidal drug carriers. Eur J Pharm Biopharm, 48:101-11.

Kanaoka E, Nagata S, Hirano K. 1999. Stabilization of aerosolized IFNgamma by liposomes. Int J Pharm, 188:165-72.

Kawashima Y, Yamamoto H, Takeuchi H, et al. 1998. Properties of a peptide containing DL lactide/glycolide copolymer nanospheres prepared by novel emulsion solvent diffusion methods. Eur J Pharm Biopharm, 45:41-8.

Kawashima Y, Yamamoto H, Takeuchi H, et al. 1999. Pulmonary delivery of insulin with nebulized DL-lactide/glycolide copolymer (PLGA) nanospheres to prolong hypoglycemic effect. J Control Rel, 62:279-87. 
Knight V, Koshkina N, Waldrep JC, et al. 2000. Anti-cancer activity of 9-nitrocamptothecin liposome aerosol in mice. Transactions of the American Clinicat and Climatological Association, 11:135-45.

Koshkina NV, Kleinerman ES, Waldrep JC, et al. 2000. 9-nitrocamptothecin liposome aerosol treatment of melanoma and osteosarcoma lung metastases in mice. Clin Cancer Res, 6:2876-80.

Lange CF, Hancock RE, Samuel J, et al. 2001. In vitro aerosol delivery and regional airway surface of liposomal cationic peptide. J Pharm Sci, 90:1641-57.

Lawrence MJ, Rees GD. 2000. Microemulsion-based media as novel drug delivery systems. Adv Drug Del, 45:89-121.

Lehr C-M. 2000. Lectin-mediated drug delivery: the second generation of bioadhesives. J Control Rel, 65:19-29.

Lemarchand C, Gref R, Passirani C, et al. 2006. Influence of polysaccharide coating on the interactions of nanoparticles with biological systems. Biomaterials, 27:108-18.

Li FQ, Hu JH, Lu B, et al. 2001. Ciprofloxacin-loaded bovine serum albumin microspheres: preparation and drug-release in vitro. J Microencapsul, 18:825-9.

Li W-I, Perzl M, Heyder J, et al. 1996. Aerodynamics and aerosol particle deaggregation phenomena in model oral-pharyngeal cavities. $J$ Aerosol Sci, 27:1269-86.

Liu FY, Shao Z, Kildsig DO, et al. 1993. Pulmonary delivery of free and liposomal insulin. Pharm Res, 10:228-32.

Lombry C, Edwards DA, Préat V, et al. 2004. Alveolar macrophages are a primary barrier to pulmonary absorption of macromolecules. Am J Physiol - Lung Cell Mol Physiol, 286:L1002-8.

Marieb EN. 2005. The respiratory system (chapter 10). In: Essentials of human anatomy and physiology, sixth edition. Benjamin-Cummings Publishing Company.

Marsh D, Bartucci R, Sportelli L. 2003. Lipid membranes with grafted polymers: physicochemical aspects. Biochim Biophys Acta-Biomembranes, 1615:33-59.

Maruyama K, Homberg E, Kennel SJ, et al. 1990. Characterization of in vivo immunoliposomes targeting to pulmonary endothelium. J Pharm Sci, 79:978-84.

Morimoto K, Katsumata H, Yabuta T, et al. 2000. Gelatin microspheres as a pulmonary delivery system: evaluation of salmon calcitonin absorption. J Pharm Pharmacol, 52:611-17.

Mu L, Feng SS. 2002. Fabrication, characterization and in vitro release of paclitaxel $\left(\right.$ Taxol $\left.^{\circledR}\right)$ loaded poly (lactic-co-glycolic acid) microspheres prepared by spray drying technique with lipid/cholesterol emulsifiers. $J$ Control Rel, 76:239-54.

Müller RH, Maassen S, Weyhers H, et al. 1996. Cytotoxicity of magnetite loaded polylactide. polylactide/glycolide particles and solid lipid nanoparticles (SEN). Int J Pharm, 138:85-94.

Müller RH, Mäder K, Gohla S. 2000. Solid lipid nanoparticles (SLN) for controlled drug delivery - review of the state of the art. Eur J Pharm Biopharm, 50:161-77.

Myers MA, Thomas DA, Straub L, et al. 1993. Pulmonary effects of chronic exposure to liposome aerosols in mice. Exp Lung Res, 19:1-19.

$\mathrm{Ng} \mathrm{K}$, Stringer KA, Cohen Z, et al. 1998. Alveolar macrophage cell line is not activated by exposure to polymeric microspheres. Int J Pharm, 170:41-9.

Niven RW, Schreier H. 1990. Nebulization of liposomes. I. Effects of lipid composition. Pharm Res, 7:1127-33.

Niven RW, Whitcomb L, Kinstler O, et al. 1994. The pulmonary absorption of aerosolized and intratracheally instilled rhG-CSF and monoPEGylated rhG-CSF. Pharm Res, 12:1343-9.

Niven RW, Whitcomb KL, Woodward M, et al. 1995. Systemic absorption and activity of recombinant consensus interferons after intratracheal instillation and aerosol administration. Pharm Res, 12:1889-95.

Omri A, Beaulac C, Bouhajib M, et al. 1994. Pulmonary retention of free and liposome-encapsulated tobramycin after intratracheal administration in uninfected rats and rats infected with Pseudomonas aeruginosa. Antimicrob Agents Chemother, 38:1090-5.
O'Riordan TG, Zwang J, Smaldone GC. 1992. Mucociliary clearance in adult asthma. Am Rev Respir Dis, 146:598-603.

Ouyang C, Choice E, Holland J, et al. 1995. Liposomal cyclosporine. Characterization of drug incorporation and interbilayer exchange. Transplantation, 60:999-1006.

Patel N, Marlow M, Lawrence MJ. 1998. Microemulsions: a novel pMDI formulation. Drug delivery to the lungs IX. Bristol, London: The Aerosol Society. p 160-3

Patton JS. 1996. Mechanisms of macromolecular absorption by the lungs. Adv Drug Deliv Rev, 19:3-36.

Patton JS, Bukar J, Nagarajan S. 1999. Inhaled insulin. Adv Drug Deliv Rev, 35:235-47.

Patton JS, Fishburn CS, Weers JG. 2004. The lungs as a portal of entry for systemic drug delivery. Proceedings of the American Thoracic Society vol 1.

Rajewski RA, Stella VJ. 1996. Pharmaceutical applications of cyclodextrins. II. In vivo drug delivery. J Pharm Sci, 85:1142-69.

Saari SM, Vidgren MT, Koskinen MO, et al. 1998. Regional lung deposition and clearance of $99 \mathrm{mTc}$-labeled beclomethasone-DLPC liposomes in mild and severe asthma. Chest, 113:1573-9.

Sakagami M, Sakon K, Kinoshita W, et al. 2001. Enhanced pulmonary absorption following aerosol administration of mucoadhesive powder microspheres. J Control Rel, 77:117-29.

Sayani AP, Chien YW. 1996. Systemic delivery of peptides and proteins across absorptive mucosae. Crit Rev Ther Drug Carrier Sys, 13:85-184.

Schanker LS. 1978. Drug absorption from the lung. Biochem Pharmacol, 27:381-5.

Schreier H, Gonzalez-Rothi RJ, Stecenko AA. 1993. Pulmonary delivery of liposomes. J Control Release, 24:209-23.

Shahiwala A, Misra A. 2004. Pulmonary absorption of liposomal levonorgestrel. AAPS PharmSciTech, 5(1)E13.

Sommerville ML, Cain JB, Johnson CS, et al. 2000. Lecithin inverse microemulsions for the pulmonary delivery of polar compounds utilizing dimethylether and propane as propellants. Pharm Dev Tech, 5:219-30.

Stern M, Ulrich K, Robinson C, et al. 2000. Pretreatment with cationic lipid-mediated transfer of the $\mathrm{Na}+\mathrm{K}+$-ATPase pump in a mouse model in vivo augments resolution of high permeability pulmonary oedema. Gene Therapy, 7:960-6.

Suarez S, O’Hara P, Kazantseva M, et al. 2001. Respirable PLGA microspheres containin- rifampicin for the $\mathrm{C}$ treatment of tuberculosis: screening in an infections disease model. Pharm Res, 18:1315-19.

Suntres ZE, Shek PN. 1998. Liposomes promote pulmonary glucocorticoid delivery. J Drug Target, 6:175-82.

Takeuchi H, Yamamoto H, Kawashima Y. 2001. Mucoadhesive nanoparticulate systems for peptide drug delivery. Adv Drug Del Rev, 47:39-54.

Taljanski W, Pierzynowski SG, Lundin PD, et al. 1997. Pulmonary delivery of intratracheally instilled and aerosolized cyclosporine A to young and adult rats. Drug Metab Dispos, 25:917-20.

Taylor KMG, Farr SJ. 1993. Liposomes for drug delivery to the respiratory tract. Drug Dev Industr Pharm, 19:123-42.

Taylor KMG, Taylor G, Kellaway IW, et al. 1990. The stability of liposomes to nebulization. Int J Pharm, 58:57-61.

Ten RM, Anderson PM, Zein NN, et al. 2002. Interleukin-2 liposomes for primary immune deficiency using the aerosol route. Int Immunopharmacol, 2:333-44.

Terzano C, Allegra L, Alhaique F, et al. 2005. Non phospholipids vesicles for pulmonary glucocorticoid delivery. Eur J Pharm Biopharm, 59:57-62.

Thomas DA, Myers MA, Wichert B, et al. 1991. Acute effects of liposome aerosol inhalation on pulmonary function in healthy human volunteers. Chest, 99:1268-70.

Vermehren C, Jørgensen K, Schiffelers R, et al. 2001. Activity of mammalian secreted phospholipase A2 from inflammatory peritoneal fluid towards PEG-liposomes. Early indications. Int J Pharm, 214:93-8. 
Vidgren M, Waldrep JC, Arppe J, et al. 1994. A study of ${ }^{99 m}$ Technetiumlabeled beclomethasone dipropionate dilauroylphosphatidylcholine liposome aerosol in normal volunteers. Int J Pharm, 115:209-16.

Waldrep JC, Arppe J, Jansa KA, et al. 1997. High dose cyclosporin A and budesonide-liposome aerosols. Int J Pharm, 152:27-36.

Waldrep JC, Gilbert BE, Knight CM, et al. 1997. Pulmonary delivery of beclomethasone liposome aerosol in volunteers. Chest, 111:316-23.

Waldrep JC, Nieminen M, Saari M, et al. 1997. Pulmonary deposition and clearance of ${ }^{99 \mathrm{~m}} \mathrm{TC}$-labeled beclomethasone liposomes in mild and severe asthmatics. Respir Crit Care Med, 155, abstract.

Wang D, Robinson DR, Kwon GS, et al. 1999. Encapsulation of plasmid DNA in biodegradable poly(D,L-lactic-co-glycolyc acid) microspheres as a novel approach for immunogene delivery. $J$ Control Release, 57:9-18.

Washington N, Washington C, Wilson C. 2001. Pulmonary Drug delivery (chapter ten). In Physiological pharmaceutics - barriers to drug absorption. Second edition. New York: Taylor and Francis.
Williams RO III, Barron MK, Alonso MJ, et al. 2000. Investigation of a pMDI system coniaining chitosan microspheres and P134a. Int J Pharm, 174:209-22.

Yamamoto A, Tanaka H, Okumura S, et al. 2001. Evaluation of insulin permeability and effects of absorption enhancers on its permeability by an in vitro pulmonary epithelial system using xenopus pulmonary membrane. Biol Pharm Bull, 24:385-9.

Zeng XM, Martin GP, Marriott C. 1995. Preparation and in vitro evaluation of tetrandrine-entrapped albumin microspheres as an inhaled drag delivery system. Eur J Pharm Sci, 3:87-93.

Zhang Q, Shen Z, Nagai T. 2001. Prolonged hypoglycemic effect of insulin-loaded polybutylcyanoacrylate nanoparticles after pulmonary administration to normal rats. Int J Pharm, 218:75-80. 
